\title{
An Incremental Algorithm for Mining Generators Representation
}

\author{
Lijun $\mathrm{Xu}$ and Kanglin Xie \\ Department of Computer Science and Engineering, Shanghai JiaoTong University, 282\#, \\ No.1954 HuaShan Road, Shanghai, China, 200030 \\ lijunxulsjtu.edu.cn, xie-kl@cs.sjtu.edu.cn
}

\begin{abstract}
This paper presents an efficient algorithm for maintaining the generator representation in dynamic datasets. The generators representation is a kind of lossless, concise representation of the set of frequent itemsets. Furthermore, the algorithm utilizes a novel optimization based on generators borders for the first time in the literature. Generators borders are the borderline between frequent generators and other itemsets. New frequent generators can be generated through monitoring them. Experiments show that our algorithm is more efficient than previous solutions.
\end{abstract}

\section{Introduction}

Frequent itemsets mining [1] is an important subject in many data mining applications, such as the discovery of association rules, correlations, sequential rules and episodes. A lot of algorithms have been proposed for this domain. But most algorithms assume that all transactions are available prior to the execution of the algorithm. However, in most cases this assumption does not hold. Many datasets are updated with blocks of data at regular time intervals. Recognizing the importance of the problem, many researchers [2-6, 11, 12] have proposed their solutions and efficient algorithms. The first incremental frequent itemsets mining algorithm, FUP, was proposed by Cheung et al. [3]. FUP2 [4] algorithm, adapted from FUP, can simultaneously handle deletions and additions. Two algorithms both adopt a level-wise search strategy like Apriori algorithm [1] and use the previous result for guiding the update. Feldman et al. [6] and Thomas et al. [11] proposed two similar algorithms respectively. The main idea of two algorithms is to keep track of frequent itemsets and the negative border that contains the itemsets form the borderline between frequent itemsets and infrequent itemsets. New frequent itemsets can be found by monitoring the negative border. Ayan et al. [2] presented UWEP algorithm, which follows the approach of FUP2. UWEP prunes the itemsets that will become infrequent by a look-ahead pruning strategy. ZIGZAG algorithm [12] is enlightened by GenMax [7] algorithm, an algorithm for discovering maximal frequent itemsets. It incrementally computes maximal frequent itemsets combining previous knowledge. But it may scan the dataset again to compute support values of some frequent itemsets that are not maximal frequent itemsets. Chi et al. [5] proposed Moment algorithm, which uses an in-memory data structure to monitor frequent closed itemsets and the itemsets that form the boundary between the frequent closed itemsets and the rest of the itemsets. Moment handles 
new transactions or deleted transactions one by one, which may cause frequent changes of the boundary and affect the performance of the algorithm.

In this paper we present an efficient algorithm, called GBorder2, to maintain the generators representation in dynamic datasets. The generators representation is a kind of lossless, concise representation of the set of frequent itemsets. The usage of the generators representation can significantly reduce the times of data scans and the number of candidates in that the generators representation can be orders of magnitude smaller than the set of all frequent itemsets. Moreover, to the best of our knowledge, the algorithm introduces a novel optimization utilizing generators borders for the first time. Generators borders are the borderline between frequent generators and other itemsets. This optimization provides significantly computational or I/O savings as new frequent generators can be generated through monitoring generators borders.

\section{Problem Definition}

Let I be a set of items. A subset $\mathrm{X} \subseteq \mathrm{I}$ is called an itemset. An itemset with $\mathrm{k}$ items is called k-itemset. Let $\mathrm{D}$ be a transactional database, where each transaction is a subset of I. The number of transactions in D is denoted by IDI. During each update, obsolete transactions are removed and new transactions are added. Let $\mathrm{d}^{+}$be the set of newly added transaction, $\mathrm{d}^{-}$be the set of deleted transactions and $\mathrm{N}$ be the updated dataset, i.e. $\mathrm{N}=\left(\mathrm{D}-\mathrm{d}^{-}\right) \cup \mathrm{d}^{+}$.

The support value of an itemset $X, \operatorname{Sup}(X)$, is the number of the transactions that contain $X$. An itemset is frequent if it satisfies the minimum support threshold $(\theta)$. Let $F$ be the set of frequent itemsets, i.e. $F=\{X|\operatorname{Sup}(X) \geq \theta| D \mid\}$.

An itemset is a generator if none of its proper subsets has the same support as it has. We denote the set of generators by $\mathrm{G}$ and the set of frequent generators by FG, i.e. $\mathrm{FG}=\mathrm{F} \cap \mathrm{G}$. Negative generators border, $\mathrm{GB}^{-}$, is defined as the set of infrequent generators whose proper subsets are frequent generators. Positive generators border, $\mathrm{GB}^{+}$, is defined as the set of frequent non-generators whose proper subsets are generators. The generators representation consists of two components: (a) FG enriched by the support value for each itemset $\mathrm{X} \in \mathrm{FG}$; (b) $\mathrm{GB}^{-}$. The following lists two important conclusions. Please refer to $[8,9]$ for more details.

Theorem 1. $\mathrm{X} \in \mathrm{G} \rightarrow \forall \mathrm{S} \subset \mathrm{X}, \mathrm{S} \in \mathrm{G} ; \mathrm{X} \notin \mathrm{G} \rightarrow \forall \mathrm{S} \supset \mathrm{X}, \mathrm{S} \notin \mathrm{G}$.

Theorem 2. Let $\mathrm{X} \subseteq \mathrm{I}$. If $\exists \mathrm{Z} \in \mathrm{GB}^{-}$and $\mathrm{Z} \subseteq \mathrm{X}$, then $\mathrm{X} \notin \mathrm{F}$. Otherwise, $\mathrm{X} \in \mathrm{F}$ and $\operatorname{Sup}(\mathrm{X})$ $=\min (\{\operatorname{Sup}(S) \mid S \in F G \wedge S \subseteq X\})$.

\section{GBorder2 Algorithm}

GBorder2 algorithm is enlightened by the idea of the negative border $[6,10,11]$. GBorder2 maintains two kinds of generators borders: $\mathrm{GB}^{-}$and $\mathrm{GB}^{+}$. $\mathrm{GB}^{-}$defines the borderline between frequent generators and infrequent generators, and $\mathrm{GB}^{+}$defines the borderline between frequent generators and frequent non-generators. Most itemsets do not change their status (from frequent to infrequent, from infrequent to frequent, from generator to non-generator or from non-generator to generator) when a 
small number of new transactions are added or a small portion of the dataset is removed. If the itemset does not change its status, nothing needs to be done except for updating its support value. Otherwise, as we shall present, the changes must come through generators borders.

Theorem 3. Let ChangedGB be a set of itemsets that belong to FG in $\mathrm{N}$ and belong to $\mathrm{GB}^{+}$or $\mathrm{GB}^{-}$in $\mathrm{D}$. If $\mathrm{X}$ is a frequent generator in $\mathrm{N}$ and is not a frequent non-generator in $\mathrm{D}$, then there exists a subset $\mathrm{Y} \subseteq \mathrm{X}, \mathrm{Y} \in \mathrm{ChangedGB}$.

Proof: There are two possible cases for $\mathrm{X}$ :

1. $\mathrm{X}$ is a frequent non-generator in $\mathrm{D}$. Let $\mathrm{Y}$ be the smallest subset of $\mathrm{X}$ that is a frequent generator in $\mathrm{N}$ but a frequent non-generator in $\mathrm{D}$. As $\mathrm{Y}$ has minimal size, all its proper subsets are frequent generators in $\mathrm{D}$. Thus $\mathrm{Y}$ belongs to $\mathrm{GB}^{+}$in $\mathrm{D}$ and $\mathrm{Y}$ belongs to ChangedGB.

2. $\mathrm{X}$ is an infrequent itemset in $\mathrm{D}$. Let $\mathrm{Y}$ be the smallest subset of $\mathrm{X}$ that is a frequent generator in $\mathrm{N}$ but an infrequent generator in $\mathrm{D}$. As $\mathrm{Y}$ has minimal size, all its proper subsets are frequent generators in $\mathrm{D}$. Thus $\mathrm{Y}$ belongs to $\mathrm{GB}^{-}$in $\mathrm{D}$ and $\mathrm{Y}$ belongs to ChangedGB.

\subsection{Algorithm Description}

The pseudo-code for GBorder2 algorithm is given in Fig. 1. We assume that each itemset $\mathrm{X}$ that belongs to frequent generators or generators borders (OldFG, OldGB or $\mathrm{OldGB}^{+}$) and its support value in $\mathrm{D}, \sup (\mathrm{X}, \mathrm{D})$, are already known.

The approach starts by scanning $\mathrm{d}^{+}, \mathrm{d}^{-}$and computing the support values of all itemsets of OldFG, OldGB ${ }^{-}$and $\mathrm{OldGB}^{+}$in $\mathrm{d}^{+}$and $\mathrm{d}^{-}$respectively (Lines 1-3). Since the addition of new transactions and the deletion of obsolete transactions, some itemsets of OldFG, OldGB ${ }^{-}$or OldGB ${ }^{+}$may change their status. Thus the frequent generators and the generators borders are determined again (Lines 4-6). ChangedGB contains the new frequent generators that originally belong to the generators borders in D (Line 7). It is used to generate candidates in the later steps.

Next, the candidates are generated and tested level by level like the classical Apriori algorithm [1] (Lines 8-26). (i+1)-candidates $\left(\mathrm{C}_{\mathrm{i}+1}\right)$, is generated based on iitemsets of ChangedGB (ChangedGB $\left.{ }_{i}\right)$, new i-generators calculated in the last whileloop steps $\left(\mathrm{G}_{\mathrm{i}}\right)$, i-generators $\left(\mathrm{NewFG}_{\mathrm{i}}\right)$ (Line 12$)$. For each candidate $\mathrm{X}$, the algorithm first determines $\operatorname{Sup}\left(\mathrm{X}, \mathrm{d}^{+}\right)$and $\operatorname{Sup}\left(\mathrm{X}, \mathrm{d}^{-}\right)$by scanning $\mathrm{d}^{+}$and $\mathrm{d}^{-}($Line 14). Then there are two possible cases when $\operatorname{Sup}(\mathrm{X}, \mathrm{D})$ is calculated. If $\mathrm{X}$ is infrequent in $\mathrm{D}$, the algorithm has to scan D and determines its support value (Line 15-16). Otherwise, its support value can be directly retrieved from OldFG according to Theorem 2 (Lines 17-18). Finally the qualified candidates are added into NewFG (Line 23), NewGB (Line 21) or $\mathrm{NewGB}^{+}$(Line 25) respectively after updating their support values.

The while-loop steps (Lines 10-26) are performed only if ChangedGB is not empty. Thus unnecessary computing and I/O requirements are avoided if there is no new generator generated. Furthermore, the number of candidates can be considerably reduced even though these steps are performed. 
Input: OldFG, OldGB ${ }^{-}, \mathrm{OldGB}^{+}, \mathrm{N}\left(\mathrm{N}=\left(\mathrm{D}-\mathrm{d}^{-}\right) \cup \mathrm{d}^{+}\right)$and $\theta$ Output: NewFG, NewGB and NewGB

1) for $\mathrm{X} \in \mathrm{O} l \mathrm{dFG} \cup \mathrm{OldGB}-\cup 01 \mathrm{dGB}^{+}$

2) $\operatorname{Scan} d^{+}, d^{-}$and calculate $\operatorname{Sup}\left(x, d^{+}\right), \operatorname{Sup}\left(x, d^{-}\right)$

3) $\operatorname{Sup}(\mathrm{X}, \mathrm{N})=\operatorname{Sup}(\mathrm{X}, \mathrm{D})+\operatorname{Sup}\left(\mathrm{X}, \mathrm{d}^{+}\right)-\operatorname{Sup}\left(\mathrm{X}, \mathrm{d}^{-}\right)$

4) $\operatorname{NewFG}=\left\{X\left|X \in O l d F G \cup O l d G B-\cup O l d B^{+} \wedge \operatorname{Sup}(X, N) \geq \theta\right| N \mid \wedge \forall S \subset X\right.$, $\operatorname{Sup}(X, N)<\operatorname{Sup}(S, N)\}$

5) $\operatorname{NewGB}^{-}=\{X|X \in O l d F G \cup O l d G B-\cup O l d G B+\wedge \operatorname{Sup}(X, N)<\theta| N \mid \wedge \forall S \subset X$, $\operatorname{SeNewFG\wedge } \forall \operatorname{S\subset X}, \quad \operatorname{Sup}(X, N)<\operatorname{Sup}(S, N)\}$

6) $\mathrm{NewGB}^{+}=\left\{\mathrm{X}\left|\mathrm{X} \in \mathrm{OldFG \cup OldGB} \cup \operatorname{OOldGB}{ }^{+} \wedge \operatorname{Sup}(\mathrm{X}, \mathrm{N}) \geq \theta\right| \mathrm{N} \mid \wedge \forall \mathrm{S} \subset \mathrm{X}\right.$,

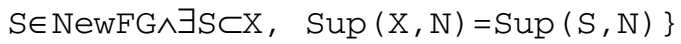

7) ChangedGB $=\left\{X \mid X \in O l d G B^{-} \cup O l d G B^{+} \wedge X \in N e w F G\right\}$

8) $n=\max \left(\left\{i \mid\right.\right.$ ChangedGB $\left.\left._{i} \neq \varnothing\right\}\right)$,

9) $\mathrm{G}_{0}=\varnothing, \quad i=0$

10) while $\left(\mathrm{G}_{\mathrm{i}} \neq \varnothing \vee \mathrm{i} \leq \mathrm{n}\right)$

11) $\mathrm{G}_{i+1}=\varnothing$

12) $C_{i+1}=\left\{X|| X \mid=i+1 \wedge \exists i\right.$-subset $S \subset X, \quad S \in C_{\text {ChangedGB }} \cup G_{i} \wedge \forall$

i-subset $S \subset X, \quad S \in N_{e w F G} \cup$ ChangedGB $\left._{i}\right\}$

13) for $\mathrm{X} \in \mathrm{C}_{\mathrm{i}+1}$

14) Scan $d^{+}, d^{-}$and calculate $\operatorname{Sup}\left(x, d^{+}\right), \operatorname{Sup}\left(x, d^{-}\right)$

15) if $\exists S \subset X \wedge S \in O l d G B$ then

16) Scan D and calculate $\operatorname{Sup}(X, D)$

17) else

18) $\quad \operatorname{Sup}(X, D)=\min \{\operatorname{Sup}(S, D) \mid S \subset X \wedge S \in O l d F G\}$

19) $\quad \operatorname{Sup}(X, N)=\operatorname{Sup}(X, D)+\operatorname{Sup}\left(X, d^{+}\right)-\operatorname{Sup}\left(X, d^{-}\right)$

20) if $\operatorname{Sup}(X, N)<\theta|N|$ then

21) Add $X$ into NewGB ${ }^{-}$

22) else if $\forall \operatorname{S\subset X}, \operatorname{Sup}(X, N)<\operatorname{Sup}(S, N)$ then

23) Add $X$ into $G_{i+1}$

24)

25) else

Add $\mathrm{X}$ into $\mathrm{NewGB}^{+}$

26) $\quad \mathrm{NewFG}=\mathrm{NewFG} \cup \mathrm{G}_{i+1}, \quad i=i+1$

Fig. 1. GBorder2 Algorithm

\subsection{Discussions}

GBorder2 handles the general case for transaction insertions as well as deletions. For the add-only case $\left(\mathrm{d}^{+} \neq \varnothing\right.$ and $\left.\mathrm{d}^{-}=\varnothing\right)$ or the delete-only case $\left(\mathrm{d}^{+}=\varnothing\right.$ and $\left.\mathrm{d}^{-} \neq \varnothing\right)$, there exists some improvements on the implementation of the algorithm.

For the add-only case, as we shall present in Theorem 4, a generator in D is still a generator in N. Then we can optimize GBorder2 by modifying Line 4-6 in Fig.1. The changes are shown in Fig. 2.

Theorem 4. Let $X$ be a generator in $D$. If $d^{-}=\varnothing$, i.e. $N=D \cup d^{+}$, then $X$ is still a generator in $\mathrm{N}$. 


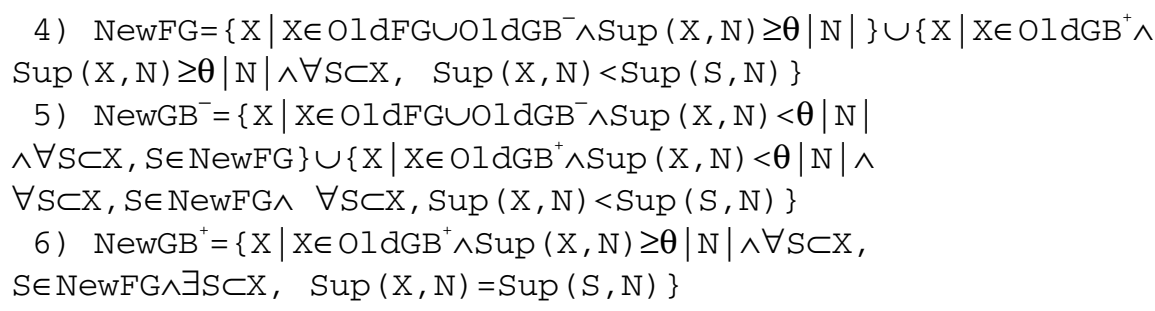

Fig. 2. Optimizations for add-only case

Proof. Let $S$ be an arbitrary subset of $X$. According the definition of generators, $\operatorname{Sup}(X, D)<\operatorname{Sup}(S, D)$. AS $S$ is a subset of $X, \quad \operatorname{Sup}\left(X, d^{+}\right) \leq \operatorname{Sup}\left(S, d^{+}\right)$. Then $\operatorname{Sup}(X, N)=\operatorname{Sup}(X, D)+\operatorname{Sup}\left(X, d^{+}\right)<\operatorname{Sup}(S, D)+\operatorname{Sup}\left(S, d^{+}\right)=\operatorname{Sup}(S, N)$.

So $\mathrm{X}$ is a generator in $\mathrm{N}$.

For the delete-only case, a non-generator in $\mathrm{D}$ is still a non-generator in $\mathrm{N}$ (See Theorem 5). So any new generator must be infrequent generator in D. We have two improvements over the pseudo-code of GBorder2. The first one is presented in Fig. 3. The second one is that Lines 15-18 are replaced with Line 16 as none of the candidates are frequent in $\mathrm{D}$.

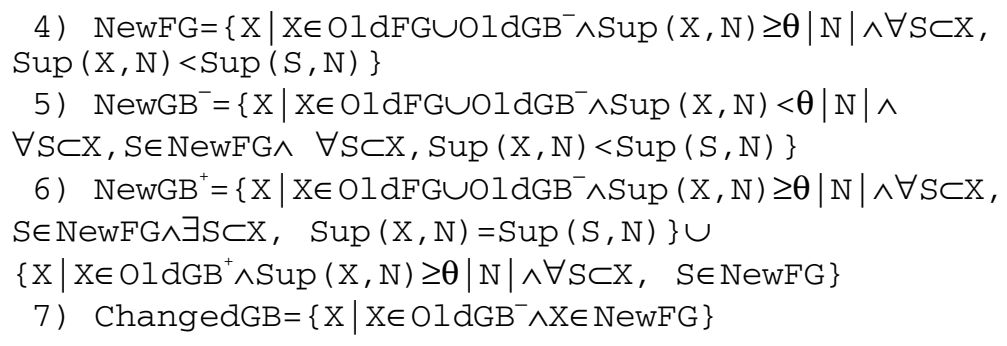

Fig. 3. Optimizations for delete-only case

Theorem 5. Let $X$ be a non-generator in D. If $d^{+}=\varnothing$, i.e. $N=D-d^{-}$, then $X$ is still a non-generator in $\mathrm{N}$.

Proof. Let $S$ be an proper subset of $X$ and $\operatorname{Sup}(X, D)=\operatorname{Sup}(S, D)$. Obviously, any transaction in $D$ that contains $S$ also contain $X . d^{-}$is a portion of $D$ and thus $\operatorname{Sup}\left(X, d^{-}\right)=\operatorname{Sup}\left(S, d^{-}\right)$. Then $\operatorname{Sup}(X, N)=\operatorname{Sup}(X, D)-\operatorname{Sup}\left(X, d^{-}\right)=\operatorname{Sup}(S, D)-\operatorname{Sup}\left(S, d^{-}\right)=$ $\operatorname{Sup}(\mathrm{S}, \mathrm{N})$. So $\mathrm{X}$ is a non-generator in $\mathrm{N}$.

\section{Experimental Results}

We performed extensive experiments to evaluate GBorder2 algorithm. We compared it with FUP2 algorithm. We implemented two algorithms using Microsoft Visual C++ 6.0. We used the same data structures and subroutines in order to minimize any performance differences caused by minor differences in implementation. The two 
algorithms are not fully optimized due to the time limitation. They were performed on a Pentium $1.2 \mathrm{G}$ processor with $1 \mathrm{G} \mathrm{MB}$, running Windows 2000.

We choose four datasets for the performance tests, which are publicly available from IBM Almaden Research Center (www.almaden.ibm.com/cs/quest/demos.html). The T10I4D100K dataset and the T40I10D100K dataset are synthetic datasets, while the connect dataset and the gazelle dataset are real-world datasets. Their characteristics are shown in Table 1.

Table 1. Characteristics of four datasets

\begin{tabular}{ccccc}
\hline Dataset & \#Items & \#Trans. & Avg. Trans. Len. & Max. Trans. Len. \\
\hline T10I4D100K & 1,000 & 100,000 & 3.7 & 31 \\
T40I10D100K & 1,000 & 100,000 & 8.5 & 77 \\
gazelle & 498 & 59,601 & 2.5 & 267 \\
connect & 130 & 67,557 & 43 & 43 \\
\hline
\end{tabular}

We first conducted several experiments to evaluate the speed up of GBorder2 over FUP2. Without loss of generality, let $|\mathrm{D}|=100 \mathrm{~K}$ and $\left|\mathrm{d}^{+}\right|=\left|\mathrm{d}^{-}\right|=10 \mathrm{~K}$. We duplicated and randomized each original dataset to obtain $110 \mathrm{~K}$ transactions. Fig. 4 shows the results over different datasets. There are two interesting trends we observe:

1) For synthetic datasets, GBorder2 shows better performance for high support thresholds than low support thresholds. The reason is that the probability of generators borders expanding is higher at low support thresholds and as a result GBorder2 may have to san the whole dataset.

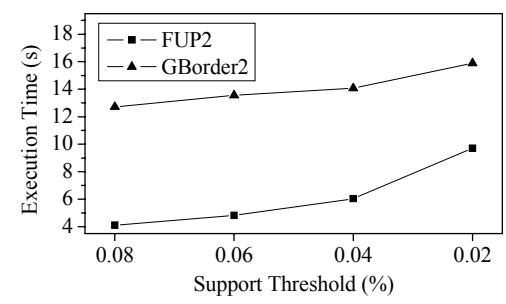

T10I4D100K

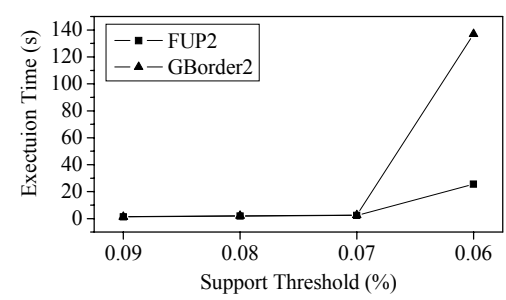

gazelle

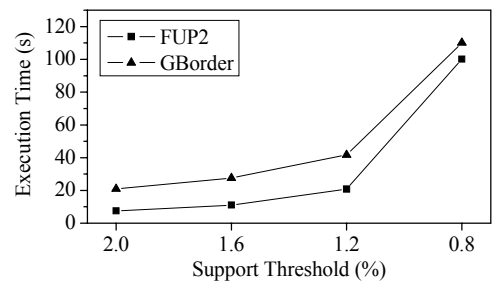

T40I10D100K

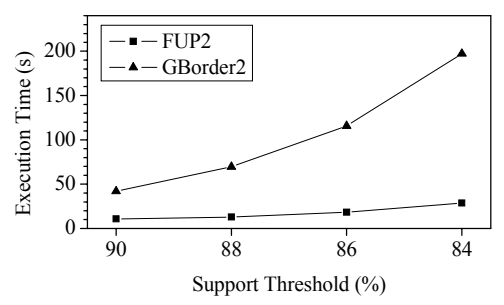

connect

Fig. 4. Performance experiments 
2) For real-world datasets, GBorder2 outperforms FUP2 throughout the entire range. Moreover, the performance gain of GBorder2 is larger for higher support thresholds. The phenomenon should be caused by the characteristics of real-world datasets. Real-world datasets are always strongly correlated datasets and a large number of frequent itemsets are non-generators for them. On the contrary, most frequent itemsets are generator for synthetic datasets.

Next, we conducted some experiments to find out if GBorder2 is able to deal with large datasets. Let $|\mathrm{D}|=\mathrm{x}$ and $\left|\mathrm{d}^{+}\right|=\left|\mathrm{d}^{-}\right|=\mathrm{x} / 10$, where $\mathrm{x}$ is varied in the experiments. We used a support threshold of $0.02 \%$ for the T10I4D100K dataset and $0.06 \%$ for the gazelle dataset. The results are plotted in Fig. 5. Obviously, the execution time of GBorder2 increases linearly as $\mathrm{x}$ increase, which implies that GBorder 2 can handle large datasets well.

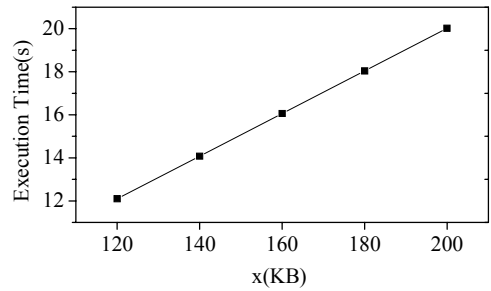

T10I4D100K

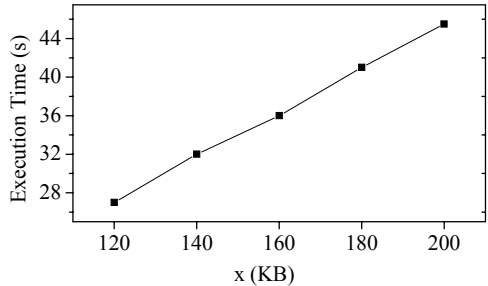

gazelle

Fig. 5. Scale-up experiments

\section{Conclusion}

The paper focuses on the problem of frequent itemsets mining in dynamic datasets. Unlike existing incremental approaches, we propose an efficient algorithm to discover the generators representation using generators borders. The generators representation is a lossless, concise representation of frequent itemsets. New frequent generators can be computed by monitoring generators borders alone. To the best of our knowledge, it is the first incremental approach that combines the border technique and the generators representation. The usage of two techniques provides significant computational and I/O savings. Extensive experimental results show the efficiency of our approach.

A number of lossless concise representations have been proposed [8]. All these representations, except for frequent closed itemsets, consist of two components: one main component and several borders. All border representations, except for the generators representation, are about two orders of magnitude more concise than frequent closed itemsets in practice. Due to the common characteristics of all border representations, our algorithm can be extended to update other border representations in an incremental manner. 


\section{Reference}

1. R. Agrawal and R. Srikant. Fast algorithms for mining association rules. In Proc. of VLDB'94 (1994) 487-499.

2. N. Ayan, A. Tansel and E. Arkun. An efficient algorithm to update large itemsets with early pruning. In Proc. of the 5th ACM-SIGKDD (1999) 287-291.

3. D. Cheung, J. Han, V. Ng and C. Y. Wong. Maintenance of discovered association rules in large databases: An incremental updating technique. In Proc. of the 12th Data Engineering (1996) 106-114.

4. D. Cheung, S. Lee, and B. Kao. A general incremental technique for maintaining discovered association rules. In Proc. of the 5th Database Systems for Advanced Applications (1997) 1-4.

5. Y. Chi, H. Wang, P. S. Yu and R. R. Muntz. Moment: maintaining closed frequent itemsets over a stream sliding window. In Proc. of ICDM'04 (2004) 59-66.

6. R. Feldman, Y. Aumann, A. Amir, and H. Mannila. Efficient algorithms for discovering frequent sets in incremental databases. In Proc. of ACM SIGMOD Workshop on Research Issues on Data Mining and Knowledge Discovery (1997) 59-66.

7. K. Gouda and M. Zaki. Efficiently mining maximal frequent itemsets. In Proc. of ICDM'01 (2001) 163-170.

8. M. Kryszkiewicz. Concise representations of association rules. In Proc. of the ESF Exploratory Workshop on Pattern Detection and Discovery (2002) 92-109.

9. N. Pasquier, Y. Bastide, R. Taouil and L. Lakhal. Pruning closed itemset lattices for association rules. In Proc. of BDA'98 (1998) 177-196.

10. H. Toivonen. Sampling large databases for association rules. In Proc. of VLDB'96 (1996) 134-145.

11. S. Thomas, S. Bodagala, K. Alsabti, and S. Ranka. An efficient algorithm for the incremental updation of association rules. In Proc. of KDD’97 (1997) 263-266.

12. A. Veloso, W. Meira Jr., M. B. de Carvalho, B. Pôssas, S. Parthasarathy, and M. Zaki. Mining frequent itemsets in evolving databases. In Proc. of SIAM'02 (2002). 